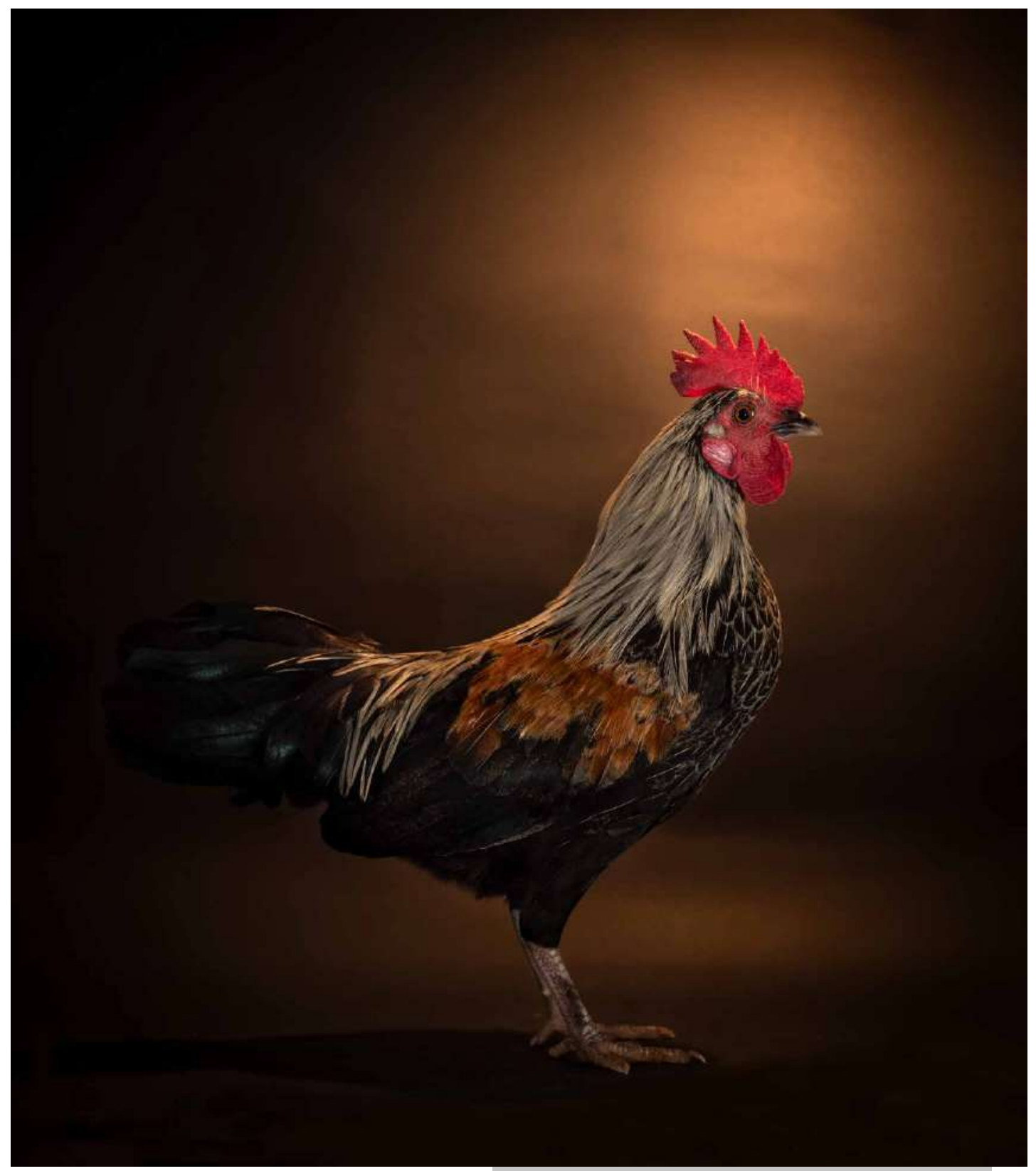

Artista invitada

Carolina Muñoz Valencia

Cubano

De la serie Retratos para entender lo esencial Fotografía digital

2020

Cortesía revista Ojo de Pez 


\title{
"La persona que me ayuda en la casa». Trabajo de cuidado doméstico remunerado, culturas y prácticas políticas en capas medias habaneras ${ }^{*}$
}

\author{
Irenia Gámez Pérez (Cuba)**
}

\section{Resumen}

La problemática de los cuidados - remunerados y no remunerados- es un tema poco presente en las esferas públicas cubanas. A partir de un estudio de caso de carácter exploratorio, cualitativo y constructivista, este artículo propone un acercamiento a las formas en que el trabajo doméstico y de cuidado remunerado se define al interior de las culturas y prácticas políticas de un grupo de pequeños y medianos propietarios(as) de negocios privados que pertenecen a las capas medias habaneras. Dicha investigación develó la feminización naturalizada del trabajo doméstico remunerado, el uso de formas de contratación verbal y la distorsión en las relaciones laborales hacia «relaciones familiares», lo cual viene acompañado de vulneración de derechos, precariedad y desamparo legal en un escenario de sombras y silencios, tanto por parte del Estado cubano como por la sociedad en general.

\section{Palabras clave}

Esfera Pública; Cultura Política; Trabajo Doméstico; Capas Medias; Cuba.

Fecha de recepción: julio de 2020

- $\quad$ Fecha de aprobación: diciembre de 2020

\section{Cómo citar este artículo}

Gámez Pérez, Irenia. (2021). «La persona que me ayuda en la casa». Trabajo de cuidado doméstico remunerado, culturas y prácticas políticas en capas medias habaneras. Estudios Políticos (Universidad de Antioquia), 60, pp. 306-324. DOI: 10.17533/udea.espo.n60a13

\footnotetext{
* Este artículo se deriva de la investigación para la tesis de maestría en Sociología Política de la Facultad Latinoamericana de Ciencias Sociales (Flacso)-Ecuador, Dinámicas entre las subjetividades sociopolíticas y las transformaciones estructurales socio-clasistas. Propietarios del sector privado en el sistema socialista cubano, 2021.

** Licenciado en Historia. Magíster en Sociología Política. Integrante del Grupo de Trabajo Populismo y República en la encrucijada neoliberal del Consejo Latinoamericano de Ciencias Sociales (Clacso). Correo electrónico: ireniagamezsofia@gmail.com - Orcid: 0000-0001-6884-6270
} 


\title{
«The Person who Helps Me at Home.» Remunerated Domestic Care Work, Cultures and Political Practices in the Middle Class of the Havana
}

\begin{abstract}
The problem of care - paid and unpaid - is a rare issue in Cuban public spheres. Based on an exploratory, qualitative and constructivist case study, this article proposes an approach to the ways in which paid domestic and care work is defined within the cultures and political practices of a group of small and mediumsized owners of private businesses that belong to the Havana middle classes. This research revealed the naturalized feminization of paid domestic work; the use of forms of verbal contracting and the distortion in labor relations towards «family relations». All of which is accompanied by the violation of rights, precariousness and legal helplessness in a scenario of shadows and silences, by the Cuban State and society in general.
\end{abstract}

\section{Keywords}

Public Sphere; Political Culture; Domestic Work; Medium Layers; Cuba. 


\section{Introducción}

En América Latina y el Caribe entre 11 y 18 millones de personas se dedican al trabajo doméstico y de cuidados remunerado, de las cuales 93\% son mujeres (ONU, OIT, CEPAL, 2020). Este tipo de trabajo se realiza, fundamentalmente, de forma precaria, genera bajos ingresos, no goza de derechos laborales y en la mayoría de los casos no existen garantías de estabilidad; eso es así en toda la región y también en Cuba. Sin embargo, este tema continúa siendo poco tratado en las esferas públicas de la mayor de las Antillas, dirimiéndose, sobre todo, en espacios informalizados y sin debate público.

Si bien existe abundante análisis al respecto en otros contextos, en la academia cubana resulta escasa la mirada sobre la contratación de trabajo doméstico y de cuidados remunerado, lo que permitiría reflexionar respecto a las representaciones simbólicas y políticas con las que se (re)construyen y «naturalizan» las asimetrías existentes en esos vínculos. ${ }^{1}$ Frente a esas ausencias, este texto se acerca al tema del cuidado desde un ángulo específico: la comprensión de las formas en que el trabajo doméstico se define al interior de las culturas políticas.

El enfoque es la exploración de los imaginarios que sobre el trabajo doméstico remunerado construyen un grupo de pequeños y medianos propietarios de negocios privados que pertenecen a las capas medias habaneras. Como se verá, los «patrones» asumen este trabajo como un intermedio entre trabajo asalariado y trabajo «familiar», hecho que distorsiona el vínculo empleador-empleada y lo «confunde» con relaciones afectivas que, la mayoría de las veces, son una cortina de humo que obscurece la necesidad de derechos laborales.

El análisis de las culturas y prácticas políticas mostrará que, de conjunto, la contratación del trabajo de cuidado doméstico remunerado es verbal, no existen derechos laborales formalizados y es tácitamente aceptado por el Estado cubano y por la sociedad en general, sin cuestionamientos ni debates en las micro y meso esferas públicas. Estas culturas y prácticas políticas no se pueden divorciar de los cambios socioeconómicos que se vienen sucediendo —en lo principal— desde la década de 1990 en la sociedad cubana, ni del

\footnotetext{
${ }^{1}$ En este sentido, se destaca el trabajo realizado por Magela Romero $(2014 ; 2019)$ sobre el trabajo doméstico en la sociedad cubana.
} 
rol protagónico de las mujeres en la economía del cuidado —remunerado o no- que se acrecienta en los actuales tiempos de crisis. Así, se imbrican la relación entre las subjetividades sociopolíticas y las transformaciones estructurales socioclasistas atravesadas por las teorías feministas del cuidado.

El objetivo principal es analizar las formas en que propietarios(as) de negocios privados en La Habana, específicamente aquellos de hospedajes para el turismo internacional, conciben el trabajo de cuidado doméstico remunerado y lo integran a su actividad económica, porque esto forma parte de sus culturas y prácticas políticas. ${ }^{2}$ De manera que el sector privado de arrendamiento turístico constituye el centro de atención y dentro de este espacio de la estructura social los dueños(as) de casas de renta son los sujetos de estudio, porque esta es una actividad que se ha convertido en auténticos negocios familiares con demanda de servicios de cuidados «directos» e «indirectos».

La Habana, por su parte, es uno de los territorios que concentra a los trabajadores del sector privado de la economía cubana (Díaz y Barreiro, 2019) e históricamente cuenta con una densidad de trabajadoras domésticas de las que «se calcula que 3.5 personas, como promedio, viven de la actividad informal por cada una que está registrada» (Martin y Capote citados en Romero 2014, p. 45); además, es el lugar que acoge a las principales instituciones políticas y culturales del país, y constituye un espacio privilegiado para la comprensión de los impactos políticos y socioestructurales derivados del periodo de crisisreformas de la década de 1990 y su posterior profundización, es decir, su territorio físico y social refleja los procesos políticos, económicos y culturales de la nación.

La fuente primaria principal de este análisis son quince entrevistas semiestructuradas con dueños(as) de negocios privados de renta en La Habana $^{3}$ y entrevistas a especialistas en temas de economía, sociología y

\footnotetext{
${ }^{2}$ Las reflexiones que siguen son parte de una investigación mayor sobre la interrelación que existe entre las subjetividades sociopolíticas y las transformaciones estructurales socioclasistas que acontecen en la sociedad cubana, en lo principal, desde inicios de 1990, las que están pensadas desde las culturas y prácticas políticas del estrato de pequeños y medianos propietarios urbanos que pertenecen a las capas medias habaneras.

${ }^{3} \mathrm{Si}$ bien en estos encuentros con dueños(as) de casas de rentas privadas se aplicó el consentimiento informado, por pedido de nuestros sujetos de estudio se usan seudónimos.
} 
culturas políticas: Dra. Dayma Echeverría, ${ }^{4}$ Dr. Rafael Hernández, ${ }^{5}$ Dr. Julio Fernández Estrada. ${ }^{6}$

Los criterios teórico-metodológicos implementados respondieron a la necesidad de analizar, sobre todo, la micro esfera pública cubana vinculada a lazos familiares, de amistad o pequeños grupos, que es donde se reproducen principalmente los pequeños y medianos negocios privados; sin excluir y menos negar la articulación que estos espacios tienen con los niveles de análisis medio y general. Concentrarse en esta dimensión se justifica porque la sociedad cubana desde la crisis de la década de 1990 ha privilegiado las zonas discursivas más «íntimas» para compartir sus desafíos, preocupaciones y expectativas cotidianas (Torres y Ortega, 2014).

De manera que esta propuesta metodológica es cualitativa y constructivista, la que se caracteriza por ser flexible y dinámica para, sin jerarquías metodológicas, responder la pregunta de investigación. Este trabajo constituye un estudio de caso de carácter exploratorio, «cuando el objetivo es examinar un tema o problema de investigación poco estudiado» (Sampieri, Fernández y Baptista, 2006, p. 100) en la sociedad cubana. Esto no quiere decir que el campo que analiza la relación entre las subjetividades políticas

[310] y los cambios socioeconómicos esté desierto, pues existen investigaciones previas como las de Rafael Hernández (2009); Ailynn Torres y Dionara Ortega (2014); Magela Romero (2014; 2019); Sara Romanò y Dayma Echeverría (2015); Daybel Pañellas (2020) que constituyen rutas teórico-metodológicas imprescindibles para abordar el tema en cuestión.

\section{Marco Teórico}

\subsection{Apuntes conceptuales de coyuntura crítica aplicados a la sociedad cubana}

Para analizar el presente del servicio doméstico remunerado en Cuba es pertinente pensar en un lapso temporal y analítico más grande que remita a la coyuntura crítica de finales de la década de 1980 e inicios de la de 1990. Se parte de la teoría de Ruth Collier y David Collier (1991) al definir la coyuntura crítica como elecciones cruciales que generan legados trascendentales, es

${ }^{4}$ Doctora en Sociología en la Universidad de La Habana. Labora en el Centro de Estudios de la Economía Cubana.

${ }^{5}$ Sociólogo, Politólogo, Filósofo e investigador. Director de la revista Temas.

${ }^{6}$ Licenciado en Derecho y en Historia. Doctor en Ciencias Jurídicas. 
decir: «un periodo de cambio significante, que sucede típicamente en distintas formas y países y que es presumida (hypothesized) para producir distintos legados» (p. 29); por otra parte, para Giobanni Capoccia y R. Daniel Kelemen (2007) la coyuntura crítica constituye «una situación en que las influencias estructurales (económica, cultural, ideológica, organizacional) sobre la acción política son significativamente distendidas» (p. 343), lo que trae consigo que la gama de elecciones posibles se expande y las consecuencias de las decisiones tomadas transcienden en el tiempo. Este concepto contiene tres elementos en interacción: el primero remite a un cambio estructural significativo; luego este cambio se manifiesta de distintas maneras según el contexto; y, por último, los cambios implementados dejan legados que transcienden en el tiempo (Collier y Collier, 1991, p. 30).

Para su comprensión se colocó de manera breve cada uno de estos elementos en la sociedad cubana actual: primero, la estructura socioclasista puede verse como «la segmentación en clases, capas y grupos - y las relaciones entre estos segmentos— (Espina, 1997, p. 83). Este tejido social en Cuba se ha reestructurado con la recomposición de capas medias, representadas fundamentalmente por el sector no estatal de la economía - propietarios, patronos, empleadores-, lo que remite a un cambio en la estructura de clases (Espina, 2008). Dichas transformaciones se basan, principalmente, en la modificación del sistema de propiedad en la que el Estado pasó de tener el «monopolio» de los medios de producción a compartirlos con un sector denominado primero no estatal — de 1993 a 2018- y luego privado —de 2019 a la actualidad-.

En el segundo elemento, dicho proceso se imbrica con coyunturas internacionales como la caída del muro de Berlín y la crisis financiera internacional (2007-2009), lo que intensificó en Cuba y en otros países socialistas el proceso de reordenamiento de su sistema político y económico. Sin embargo, los cambios estructurales implementados en la mayor de las Antillas no son réplica de lo acontecido en otros países socialistas ${ }^{7}$ y con ello se identifica el segundo elemento del concepto de coyuntura crítica, el cual se refiere a las distintas maneras en que se manifiesta el cambio según el contexto.

\footnotetext{
${ }^{7}$ Se trata de China, Vietnam y Cuba, solo para mantener el análisis en el marco de los países con modelos de desarrollo socialista, pero esta crisis fue mucho más abarcadora y afectó a una multiplicidad de naciones.
} 
El tercer elemento remite a legados difíciles de revertir y para ceñirse al tema se realizará un rápido recorrido constitucional-jurídico por el trabajo «por cuenta propia» en general y el trabajo doméstico remunerado en particular, el cual se ha ampliado en la sociedad cubana durante los últimos veinte años.

Mediante el Decreto Ley 141 de $1993^{8}$ se estimuló el trabajo por cuenta propia, lo que creó técnicamente la figura de la propiedad privada (Aquino, Cruz, Guanche y Hernández, 2015, p. 112). Este camino continuó hacia la llamada «Actualización del modelo socioeconómico» ${ }^{9}$ y tiene su punto cúspide en la aprobación — por referéndum — en 2019 de una Constitución que reconoce la propiedad privada, lo que constituye uno de los legados de la década de 1990 difíciles de revertir. En este contexto, la actividad de «personal doméstico» fue la número 32 de las aprobadas en la reforma de 1993 y a partir de ese momento se desincrusta esta labor del sector estatal.

\subsection{Pequeños y medianos propietarios de casas para el turismo internacional y trabajadoras domésticas}

Los trabajadores, por cuenta propia, forman parte del sector privado de la economía cubana y se definen «esencialmente por su condición laboral [312] independiente [del Estado» (Hernández y Domínguez, 2013, p. 23). Dentro de este sector hay un amplio espectro de personas que ocupan diferentes posiciones en la estructura social, una parte de ellas conforman la capa media y esta última está configurada por diferentes estratos —asalariados, profesionales independientes, pequeños y medianos empleadores, entre otros-. Este análisis se acerca solo a uno de esos estratos: pequeños y medianos propietarios(as) de casas de renta para el turismo internacional que residen en La Habana.

Las trabajadoras domésticas, por su parte, fueron destinatarias de programas educativos y laborales implementados en la década de 1960, los cuales estimulaban el abandono de este tipo de responsabilidad laboral, lo que trajo como resultado que una década más tarde el discurso público oficial promulgara «el fin de las domésticas» (Romero, 2014, p. 29) en el país. No obstante, y bajo las sombras, un grupo reducido permaneció

\footnotetext{
${ }^{8}$ Resolución conjunta núm. 1 del Comité Estatal de Trabajo y Seguridad Social y del Comité Estatal de Finanzas por la que se adoptan disposiciones sobre el trabajo por cuenta propia (N. A).

${ }^{9}$ Este documento se elaboró según los lineamientos aprobados en el 6to. y 7 mo. congresos del Partido Comunista de Cuba realizados en 2011 y 2016, respectivamente, y afectó al modelo político-económico de la sociedad cubana de forma multidimensional (N. A).
} 
desarrollando estas labores, y tanto para las que lo realizaban como para quienes las contrataban existía un cierto rechazo bajo la visión de que «este tipo de empleo, solo podía existir en un país subdesarrollado y de explotación social» (p. 31).

Para esconderse de esta no-aprobación pública se creó el eufemismo «la compañera que ayuda en la casa», lo que poco a poco se fue convirtiendo en lo que ahora es «la persona que me ayuda». De acuerdo con James Scott (1990, p. 78): «siempre que en el lenguaje nos encontramos con un eufemismo significa, casi infaliblemente, que nos hemos topado con un tema delicado». Al utilizar eufemismos, lo que se intenta es mostrar «un rostro inocuo a una actividad o hecho que muchos podrían considerar moralmente ofensivo» ( $p$. 79).

\subsection{Capas medias, culturas y prácticas políticas}

Desde inicios de 1990 en la sociedad cubana vienen desarrollándose reformas económicas que han generado la recomposición acelerada del tejido y la estructura social (Burchardt, 1998; Espina, 2003). Esto ha producido una reestratificación, entendida como:

Un proceso de cambio de una estructura social dada (regional, nacional, territorial, local) que se caracteriza por el ensanchamiento de las distancias económicas y sociales entre los componentes de dicha estructura, por la aparición de nuevas clases, capas y grupos sociales que generan nuevas diferencias sociales o expanden las ya existentes, por la diversificación de las fuentes de ingresos y la polarización de estos, lo cual torna más evidente y palpable la existencia de una jerarquía socioeconómica, de un arriba y un abajo en la estructura social asociado a las diferencias en la disponibilidad económica y en las posibilidades de acceso al bienestar material y espiritual (Espina, 2008, p. 161).

Este proceso visibilizó capas medias en Cuba que se diferencian de otras capas sociales por «la posesión de activos significativos» (Espina, 2020, p. 111), los que en el caso de estudio se materializan con la propiedad o administración de una o varias viviendas dedicadas a la renta para el turismo internacional.

Otro rasgo es la «capacidad para generar ingresos monetarios directos o el acceso a bienes y servicios» (Espina, 2020, p. 111). El alojamiento al turismo 
internacional es un tipo de servicio que posibilita ingresos inmediatos en una relación propietario-cliente, sin necesidad de acudir a terceros; además, las ganancias económicas de este grupo social se diseminan con otros tipos de servicios, lo que para los sujetos de estudio se traduce en la obtención de recursos económicos complementarios, resultado de ofrecer alimentos, transporte, peluquería, entre otros servicios.

A estas particularidades se suma que comparados con otras capas sociales de menor capacidad económica pueden «incrementar el consumo, diversificar y mejorar satisfactores de necesidades básicas y no básicas» (Espina, 2020, p. 111). Si bien este planteamiento se basa en diferencias de recursos económicos entre las diferentes capas sociales que conforman la estructura social cubana y marca fronteras entre lo primordial o no, algo que es cuestionable según lo imprescindible para cada cual, lo cierto es que llama la atención sobre el aumento y diversificación del consumo por parte de las capas medias cubanas en relación con otras que cuentan con menores recursos económicos.

Ahora bien, en este reacomodo no solo cambian las formas de producir, sino que al mismo tiempo se pueden reconfigurar las culturas y prácticas [314] políticas, entendidas las primeras como «los complejos vínculos que se tejen entre la esfera pública, la vida política y los universos o representaciones que sobre ésta poseen los miembros de toda comunidad» (Morán, 1999, p. 98); por otra parte, las prácticas políticas se refieren a espacios dinámicos de aprendizajes donde se (re)construyen valores y normas de conducta, de manera que conforman «auténticos locus de aprendizajes, de atribución de significados y de elaboración de estrategias y repertorios de acción social» (Cefaï, 2001 citado en Benedicto y Morán, 2002, p. 77). Para esto se tiene en cuenta que entre los principales lugares de sociabilidad señalados por María Luz Morán (2003) se encuentran el entorno familiar —en especial «nuclear»—, puesto que allí se «moldean» la «personalidad ciudadana» con la que los individuos desarrollan su vida política (p. 41). Se alude, entonces, a la capacidad que tiene la agencia para apropiarse y transformar por medio de sus prácticas políticas y discursivas, los componentes de sus culturas políticas articuladas, en especial, a contextos de cambios estructurales.

Por tanto, las reconfiguraciones que se producen entre esfera pública, vida política y las representaciones que sobre estas dos tienen los sujetos se expresan en distintas dimensiones: microesferas — familia, amigos o 
un reducido grupo de personas con intereses comunes-; mesoesferas medios de comunicación dentro del Estado-nación-; y macroesferas conglomerados comunicativos regionales o mundiales - con «fronteras que nunca son definitivamente borradas» (Keane, 1997, p. 73). El análisis de cada una de ellas para abordar las subjetividades y culturas políticas aportan a la comprensión del conjunto del proceso.

Desde la caída del muro de Berlín en la población cubana se verificó un retorno a los espacios más «íntimos» y un «retraimiento» hacia la familia como espacio casi único de preocupación y satisfacción de los sujetos, y como anclaje social trascendental (Martin y Perera citados en Torres y Ortega, 2014, p. 64). Esta microesfera pública constituye un lugar «casi invisible» (Keane, 1997, p. 60) y privilegiado para analizar los «discursos ocultos» que sostienen, desde las culturas y prácticas políticas, procesos visibles en la economía, la sociedad y la política en general.

Con «discursos ocultos» se refiere a conductas «fuera de escena» (Scott, 1990, p. 28), con las cuales se elaboran tanto estrategias de reproducción del poder como mecanismos de resistencias para reducir los efectos de dicho poder. Estos discursos ocultos se conectan con los microespacios de la esfera pública y los imaginarios que proyectan allí los sujetos de estudio sobre su vida política. En este orden analítico interesa explorar aquellas zonas conformadas por personas con cercanías afectivas o intereses compartidos que constituyen lugares ideales para develar representaciones sociales «ocultas».

Ahora bien, estos discursos ocultos deben observarse en relación con los discursos públicos, lo que remite a las «apariencias», la imagen que sobre sí mismas proyectan las personas y los grupos sociales, es decir, la construcción de un «autorretrato» (Scott, 1990, p. 43). Otra arista de este análisis son las zonas de incesante conflicto que se generan entre los discursos públicos y ocultos, pues allí «el discurso oculto presiona y pone a prueba los límites de lo que se puede decir sin correr ningún riesgo» (p. 197). A estos espacios conflictivos James Scott (1990) los denomina «infrapolítica», la que con formas simples y casi imperceptibles de manifestarse constituyen los cimientos de acciones políticas futuras. Dicho de otra manera, si se rastrea en reversa acciones políticas que se enfrentan abiertamente al poder, se encontrará en los rumores, los chismes, cuentos populares, canciones y chistes las raíces ideológicas de aquella confrontación abierta (Scott, 1990). Es importante aclarar que en este terreno de la infrapolítica no se realizan 
demandas públicas ni se disputan de manera explícita espacios de poder, en su lugar prevalece el disfraz y el anonimato, donde «las cosas no son como parecen» (p. 235).

\subsection{Aproximación a teorías feministas sobre el trabajo de cuidado doméstico}

Se entiende el género como "contenedor" de comportamientos, mandatos y aspiraciones, que rigen la vida de las personas según su sexo» (Martínez y Voorend, 2009, p. 6). Los estudios feministas han analizado de forma amplia el campo del trabajo de cuidados y doméstico, tanto remunerado como no remunerado. En ese marco se encuentran una variedad de conceptos: economía del cuidado, cuidado social, regímenes de cuidado, el diamante del cuidado, entre otros. En esta investigación se asume el cuidado como «una función social que integra actividades, bienes y relaciones destinadas al aseguramiento de la vida y a la búsqueda de bienestar de las personas [...] por tanto involucran tanto activos económicos como recursos emocionales y valores morales» (Rico y Robles citados en Torres, 2020, p. 9); es decir, constituye una (re)producción material, relacional y espiritual que implica corresponsabilidad social.

El cuidado, en tanto relación entre las personas que lo brindan y las que lo reciben, no es «privado», sino que está atravesado por lo «social» en cuanto a género, clase y por las políticas «públicas» que lo (re)constituyen (Esquivel, 2011; 2013). Entonces, el trabajo doméstico y de cuidado remunerado implica recursos monetarios; derechos y deberes; se puede desarrollar por cuenta propia, de manera formal o informal; su principal escenario son los hogares; se puede interpretar como «cuidado indirecto» en relación con las labores domésticas — limpiar, cocinar-y «directo» cuando incluye atención a personas «dependientes» —niños(as) y ancianos(as)—. Su análisis permite acercarse a propiedades y sensibilidades generales de los acuerdos sociales.

Las personas que históricamente se han dedicado al trabajo doméstico remunerado son mujeres afectadas transversalmente por diferentes tipos de discriminaciones — raza, género, clase social, entre otros-, esto deriva en una invisibilidad social que retrasa la posibilidad de situarse en las agendas públicas de los gobiernos y la (re)construye culturas y prácticas políticas que subvaloran el trabajo realizado por ellas. En la sociedad cubana esta situación se (re)produce y tiene mayor incidencia social a partir de la crisis de la 
década de 1990, porque los cambios estructurales que vienen aconteciendo desde entonces fortalecen diferentes tipos de desigualdades sociales y, entre otros, el binomio de mujeres empobrecidas-mujeres de éxito. Las primeras se mantuvieron o empezaron a formar parte de la organización social del cuidado como proveedoras de estos servicios y las segundas pasaron al sector privado, cooperativo o de inversión extranjera como receptoras de estos. Esto (re)genera relaciones asimétricas de poder con contenidos y prácticas políticas que modifican las identidades sociales (Fraser y Lamas, 1991).

De manera que para lograr reconocimiento en este sector laboral, reducción y redistribución de los cuidados entre los diferentes agentes sociales y representación política de las personas que realizan cuidado remunerado se debe entender y analizar de forma crítica las subjetividades que mantienen a las trabajadoras domésticas en las «sombras», pues estas prácticas y culturas políticas están articuladas a la presión social, lo que permite unir fuerzas para romper el cordon saniterie (Scott, 1990) trenzado alrededor de este tema o, en su lugar, sostenerlo en el tiempo.

\section{Culturas y prácticas políticas de un grupo social que pertenece al estrato medio habanero, en relación con el trabajo de cuidado doméstico remunerado}

Se comienza por explorar los imaginarios que le otorgan los sujetos de estudio al significante «política» porque este construye ideas que justifican la legitimidad de sus actos, de forma tal que parecen «naturales». Se encuentra que esta palabra puede traducirse en «La defensa de la Revolución, de lo que se ha logrado» (Mercedes, comunicación personal, 29 de abril, 2019). Para otras de las personas entrevistadas constituye algo negativo que obstaculiza su desarrollo en el mundo privado: «A la larga, es lo que nos sigue jodiendo la vida [...] yo no quiero saber nada de política, yo quiero buscarme los pesos» (Armando, comunicación personal, 5 de mayo, 2019); «No me gusta y no creo» (Iris, comunicación personal, 6 de mayo, 2019). En contraste, se encuentran las personas que visualizan la política como «El centro del debate, nuestra preocupación por Cuba, es todo» (Paula, comunicación personal, 26 de abril, 2019); «Importante, fundamental, diría yo» (Indira, comunicación personal, 22 de abril, 2019).

Este diapasón, que en unos casos identifica la palabra política con la construcción de dos entidades discursivas centrales: «Revolución y Pueblo» 
(Corrarello, 2016, p. 16), en otros muestra una mirada que pondera el mercado por encima de las políticas sociales y una tercera que proyecta pensamiento crítico donde las relaciones mercantiles tienen que estar articuladas con derechos políticos y ciudadanos, construyen diferentes tipos de ciudadanías. Al mismo tiempo, estas formas de «ser ciudadano» asumen de maneras diferentes la relación con las trabajadoras domésticas. En el primer caso se encuentra la única persona del grupo que planteó no tener a nadie realizando esta labor y que sobre los integrantes de su gremio aseguró: «Hay una tendencia de que han pasado a otro estrato y quieren tener sirvientes» (Mercedes, comunicación personal, abril 29, 2019). Dicha expresión equipara a las trabajadoras domésticas con sirvientes y, por lo tanto, no reconoce este tipo de trabajo como una forma digna de sostener las necesidades vitales, lo que tiene conexión con las culturas políticas de las décadas de 1960 y 1970 que escondieron esta labor de la luz pública.

Mientras, los ciudadanos con tendencias neoliberales y aquellos que identificamos como «críticos o reflexivos» coinciden en contratar de manera informal a trabajadoras domésticas. Sobre este particular una propietaria de casa de renta comenta que a pesar de no establecer contratos «no hemos tenido ningún incidente» (Paula, comunicación personal, abril 26, 2019). Sobre esta práctica que prevalece en el sector privado de la economía cubana, el profesor de Derecho Julio Fernández Bulté explica:

La mayoría de los negocios están en las casas de los dueños, donde puede que ocurran abusos cotidianos o los trabajadores se dieron cuenta que están siendo explotados y deciden hacer una huelga de brazos caídos. En ese momento el dueño puede romper el contrato verbal de forma igualmente verbal y les dice que ya no son sus trabajadores por lo que tienen que abandonar su casa o puede llamar a la policía y acusarlos de violación de domicilio. O sea, no hay para ellos ningún derecho de reclamo o protesta (comunicación personal, 9 de mayo, 2019).

Es decir, los trabajadores subordinados en el sector privado de la economía cubana son un estrato vulnerable y en el caso de las trabajadoras domésticas esta vulnerabilidad aumenta por la situación laboral en que se encuentran, en la que las relaciones contractuales se sustituyen por «familiares»: «Ella es como de la familia [...] es como a un miembro más de la familia, su mamá también trabajaba aquí [...] nos ayudamos mutuamente» (Iris, comunicación personal, 6 de mayo, 2019). 
Aquellos(as) que tienen una posición más reflexiva señalan que la Constitución política aprobada el 10 de abril de 2019 sobre el tema del cuidado fue «una oportunidad que se perdió, porque quedaron vacíos, ausencias, como cuidado infantil, salario no remunerado» (Paula, comunicación personal, 26 de abril, 2019). Sin embargo, estos ciudadanos(as) propietarios(as) también mantienen a las trabajadoras domésticas en la informalidad y sin derechos constituidos. Si se tiene en cuenta que desde 2018 los arrendatarios de vivienda fueron reconocidos como personas jurídicas y pueden determinar «qué contratar, con quién contratar y cómo contratar, además de cuándo hacerlo» (Soto, 2019, p. 253), resulta incoherente su discurso político con las prácticas políticas que desarrollan en relación con las personas que realizan cuidados domésticos.

¿Por qué no reconocer a las personas que realizan trabajo doméstico en su condición de trabajadoras con deberes y derechos? ¿Por qué el Estado cubano y la sociedad civil no estimulan el debate en la mesoesfera pública sobre las condiciones laborales de las trabajadoras domésticas? ¿Qué factores inciden para las escasas políticas públicas dirigidas hacia este sector laboral?

\subsection{Trabajo doméstico y de cuidado. Cultura tributaria}

Una de las posibles respuestas a estas preguntas se basa en las culturas y prácticas tributarias desarrolladas en la sociedad cubana. ${ }^{10}$ En la actualidad, la evasión fiscal es un problema que afecta a la región y Cuba no es una excepción, donde las mayores indisciplinas tributarias — hechas públicasse reportan en el pago del impuesto sobre los ingresos personales de los trabajadores por cuenta propia. Según cifras oficiales, en 201468519 privados subdeclararon $(47,7 \%)$ y al año siguiente lo hicieron 79359 (55\%) (Arrocha y Proenza, 2019, p. 234).

Este escenario puede tener sus raíces desde finales de la década de 1960, ya que la mayoría de la población cubana no tuvo que hacer declaraciones de impuestos sobre los ingresos personales, hasta que en 1994 se implementó un sistema tributario que incluía una amplia gama de contribuciones del sector privado. Esto ha derivado en una cultura tributaria y un grado de desarrollo de las instituciones dedicadas a este fin muy elemental, a lo que se suma que

\footnotetext{
${ }^{10}$ El impuesto constituye un aporte monetario que va desde la población hacia el Estado para satisfacer necesidades colectivas de un país, lo que implica, como uno de sus indicadores, la cultura tributaria de los contribuyentes (N.A).
} 
no está presente de forma sistemática en la mesoesfera pública los debates sobre el uso de los presupuestos institucionales y las personas, en general, no dominan la forma en que sus impuestos son ejecutados. Al respecto, la visión del grupo estudiado se refleja en testimonios como: «nuestra cultura legal es escasa» (Armando, comunicación personal, mayo 5, 2019); «Chica, yo sé que lo que aportamos es beneficio para todos [salud, educación], pero podríamos hacer cosas más tangibles en el barrio donde vivimos y de eso no tendría que ocuparse el Estado» (Ivis, comunicación personal, mayo 8, 2019).

Para 2018 la República de Cuba ocupó el puesto 61 del Índice de percepción de la corrupción entre 180 países (Transparency International, 2019). Esto devela que existen parámetros aceptables de confianza en el uso que el Estado cubano le da a los fondos recaudados mediante los impuestos. Sin embargo, se muestran relaciones conflictivas entre los montos de recaudación y el uso de los impuestos aplicados al sector privado de la economía. Esta conflictividad se expresa no solo en la evasión de pagos de impuestos, sino también en los procesos de configuración de las identidades, prácticas y culturas políticas. Como plantea Sofia Argüello (2013), los espacios sociales conflictivos conforman procesos configurativos que dejan huellas normativas y pueden establecer la emergencia de actores sociales que cuestionan y subvierten los órdenes constituidos. Estas relaciones conflictivas forman parte de un proceso activo entre estructuras y agencias que se sustentan en pautas culturales, en relaciones de fuerza y en estructuras emocionales preexistentes.

Sobre las culturas y prácticas políticas que tienen las trabajadoras domésticas surgen cuestionamientos no contemplados en esta investigación que pueden abrir paso a estudios futuros: ¿Cómo visualizan las empleadas domésticas en Cuba a las personas con las que tienen un «pacto» laboral y que reciben los beneficios de su trabajo? ¿Por qué aceptan realizar trabajo doméstico sin contrato escrito? ¿Qué marco legislativo protege a las trabajadoras domésticas remuneradas en Cuba? Estos son temas pendientes, que vale la pena explorar para tener una visión de conjunto.

\section{Reflexiones finales}

Se puede plantear que en Cuba, al igual que en el resto del mundo, el sector de cuidados está feminizado, subvalorado e invisibilizado, y esta situación se sostiene, entre otros factores, por las culturas y prácticas políticas de los ciudadanos(as), articuladas a las políticas públicas del gobierno en la isla. El binomio trabajadora doméstica-familia suprime los derechos laborales 
de las primeras, justifica el hecho de no realizar contrato escrito y permite eludir el pago de impuestos por recibir este tipo de servicio laboral.

Los pequeños y medianos propietarios(as) habaneros de casas de renta para el turismo internacional investigados proyectan universos políticos diversos $y$, en ocasiones, contradictorios cuando se imbrican al cuidado doméstico remunerado, pero en todos los casos se aprecia un no reconocimiento de esta labor y una coartación de los derechos laborales para las personas que la realizan.

Dentro de esta visión general se proyectan diferentes formas de manifestarse las subjetividades políticas. Se identificaron a los que no emplean a trabajadoras domésticas remuneradas y las perciben como «sirvientes», por lo tanto, no reconocen esta labor como una forma digna de sostener las necesidades vitales de ellas y de sus familias. Esta proyección da continuidad a las culturas y prácticas políticas establecidas en la sociedad cubana desde finales de la década de 1960 que «satanizaron» este tipo de empleo. Estos ciudadanos(as) son fieles a los legados que dejaron las reformas realizadas, sobre todo, en los primeros años del proceso de la Revolución cubana, lo que supera las carencias materiales y reacomodos estructurales que vienen aconteciendo desde la década de 1990. Para estos casos no es posible establecer una relación directa entre cambios socioestructurales y cambios en los universos sociopolíticos.

En otro grupo están los que asumen a estas trabajadoras como «parte de la familia», y dentro de él se encuentran los que exponen culturas y prácticas políticas asociadas a tendencias neoliberales al solicitar mayor participación del sector privado en la economía y no en la política. También se encuentran aquellos(as) que muestran aspiraciones no resueltas en la nueva constitución relacionada con el trabajo de cuidado, lo que entra en contradicción con sus prácticas políticas. Estos ciudadanos(as) señalan fisuras, debilidades y desacuerdos que todo sistema posee y que, en ambos casos, suprimen los derechos laborales para las trabajadoras domésticas.

Todo ello devela tanto permanencia de culturas y prácticas políticas desarrolladas en la década de 1960 como (re)configuraciones resultado de los legados dejados por la coyuntura crítica de la década de 1990. Esta situación está marcada por el escaso debate sobre el trabajo de cuidado remunerado o no- en la mesoesfera y su retraimiento a discursos ocultos en 
la microesfera. En tal escenario resulta imprescindible tanto el debate público sobre el trabajo doméstico y de cuidado como elaborar políticas con derechos universales que tengan en cuenta al invisibilizado sector de las trabajadoras domésticas en la sociedad cubana.

\section{Referencias bibliográficas}

1. Aquino, Ulises; Cruz, Orlando; Guanche, Julio César y Hernández, Rafael. (2015) Veinte años: la reforma constitucional (1992-2012). Temas, 81-82, pp. 111-123.

2. Argüello Pazmiño, Sofía. (2013). El proceso de politización de la sexualidad: identificaciones y marcos de sentido de la acción colectiva. Revista Mexicana de Sociología, 75 (2), pp. 173-200.

3. Arrocha Hernández, Claudia y Proenza González, Dayané. (2019). La evasión tributaria: factores influyentes en su aparición. Un estudio en transportistas del municipio 10 de octubre. En: Tabares Neyra, Lourdes (coord.). Retos de la Administración Pública hoy: en el marco del perfeccionamiento del modelo económico cubano (pp. 231-259). La Habana: Editorial Universitaria.

4. Benedicto, Jorge y Morán, María Luz. (2002). La construcción de una ciudadanía activa entre los jóvenes. Madrid: C/José Ortega y Gasset.

5. Burchardt, Hans Jürgen. (1998) ¿Deberían leer en Cuba a Bourdieu? Socialismo, estructura social y capital social. Análisis Político, 34, pp. 26-46.

6. Caballero, Claudia M. y Pañellas Álvarez, Daybel. (2014). Cuentapropismo y redes sociales. Otras evidencias de heterogeneidad social en Cuba. Alternativas Cubanas en Psicología, 2 (5). http://acupsi.org/articulo/64/cuentapropismo-y-redessociales-otras-evidencias-de-heterogeneidad-social-en-cuba.html

7. Capoccia, Giovanni y Kelemen, R. Daniel. (2007). The Study of Critical Junctures. Theory, Narratives and Contrafactuals in Historical Institutionalism. World Politics, 59 (3), pp. 341-369. https://doi.org/10.1017/S0043887100020852

8. Collier, Ruth y Collier, David (1991). Shaping the Political Arena. Critical Junctures, the Labor Movement, and Regimen Dynamics in Latin America. Princenton: Princenton University.

9. Corrarello, Ana María. (2016). Adecuación estratégica en el Discurso de Fidel Castro de la etapa fundacional al proyecto socialista soviético (1963-1989) Un análisis retorico-discursivo. FILO UBA. http://repositorio.filo.uba.ar/bitstream/handle/ filodigital/2826/uba_ffyl_t_2016_se_corrarello.pdf?sequence $=1$ \&isAllowed $=y$

10. Cuba. Consejo de Estado. Decreto Ley 141 (9 de agosto de 1993). Gaceta Oficial, núm. 5 extraordinario, pp. 11.

11. Cuba. Referéndum. Constitución. (10 de abril de 2019). Se reconocen como una de las formas de propiedad: d) privada: la que se ejerce sobre determinados medios de producción por personas naturales o jurídicas cubanas o extranjeras; con un papel complementario en la economía. Gaceta Oficial. Art. 22. 
«La persona que me ayuda en la casa». Trabajo de cuidado doméstico remunerado...

12. Díaz Fernández, Ileana y Barreiro Pousa, Luis. (2019). Un análisis del sector cuentapropista en La Habana. Economía y Desarrollo, 161 (1). http://www. econdesarrollo.uh.cu/index.php/RED/article/view/629

13. bEsquivel, Valeria. (2011). La economía del cuidado en América Latina: poniendo a los cuidados en el centro de la agenda. El Salvador: PNUD.

14. Esquivel, Valeria. (2013). El cuidado en los hogares y las comunidades. Documento conceptual.

15. Espina Prieto, Mayra Paula. (1997). Transformaciones recientes de la estructura socioclasista cubana. Papers 52, pp. 83-99. https://ddd.uab.cat/pub/papers/02102862 n52/02102862n52p83.pdf

16. Espina Prieto, Mayra Paula. (2003). Reajuste y movilidad social en Cuba. Clacso. http://biblioteca.clacso.edu.ar/ar/libros/cuba/cips/caudales05/Caudales/ ARTICULOS/ArticulosPDF/1117E018.pdf

17. Espina Prieto, Mayra Paula. (2008). Políticas de atención a la pobreza y la desigualdad. Examinando el rol del estado en la experiencia cubana. Buenos Aires: Clacso.

18. Espina Prieto, Mayra Paula. (2020). Reforma y emergencia de capas medias en Cuba. Revista Nueva Sociedad, 285, pp. 108-121.

19. Fraser, Nancy y Lamas, Marta. (1991). La lucha por las necesidades: esbozo de una teoría crítica socialista-feminista de la cultura política del capitalismo tardío. Debate Feminista, 3. https://doi.org/https://doi.org/10.22201/cieg.2594066xe.1991.3.1493

20. Hernández, Rafael. (2009). El año rojo. Política, sociedad y cultura en 1968. Revista de Estudios Sociales, 33, pp. 44-54. https://doi.org/10.7440/res33.2009.03

21. Hernández, Rafael y Domínguez, Jorge I. (coords.). (2013). Cuba, la Actualización del Modelo. Balance y perspectiva de la transición socialista. La HabanaWashington, D. C.: Temas y David Rockefeller Center for Latin American Studies. https://drclas.harvard.edu/files/drclas/files/cuba_la_actualizacion_del_modelo.pdf

22. Keane, John. (1997). Transformaciones estructurales de la esfera pública. Estudios Sociológicos del Colegio de México, xv, 43, pp. 47-77.

23. Morán, María Luz. (1999). Los estudios de cultura política en España. REIS, 85, pp. 97-129. https://doi.org/10.2307/40184101

24. Morán, María Luz. (2003). Aprendizajes y espacios de la ciudadanía para un análisis cultural de las prácticas sociopolíticas. Íconos, 15, pp. 31-44.

25. Martínez Franzoni, Juliana y Voorend, Koen. (2009). Sistemas de patriarcado y regímenes de bienestar en América Latina. ¿Una cosa lleva a la otra? Fundación Carolina Documento de Trabajo, 37.

26. ONU Mujeres; OIT y Cepal. (2020). Trabajadoras Remuneradas del hogar en América latina y el Caribe frente a la crisis del COVID-19. Cepal. https://repositorio. cepal.org/bitstream/handle/11362/45724/1/Informe_CEPAL-ONUmujeres-OIT_es.pdf

27. Pañellas, Daybel. (2020). Grupos e identidades sociales en cambio. Novedades en Población, 16 (31), pp. 64-84. 
28. Romanò, Sara y Dayma Echeverría. (2015). Movilidad social y cuentapropismo: reflexiones sobre un estudio empírico en Cuba. Temas, 84, pp. 37-44.

29. Romero Almodóvar, Magela. (2014). De lo simbólicamente exacto a lo simbólicamente verdadero. Domésticas y revolución en Cuba: entre cambios y desafíos. Clacso. http://biblioteca.clacso.edu.ar/clacso/becas/20141128035630/ ensayomagelaromero.pdf

30. Romero Almodóvar, Magela. (2019). Género, cuidado de la vida y política social en cuba. Estrategias, actores y recomendaciones para una mayor corresponsabilidad. Friedrich Ebert Stiftung. http://library.fes.de/pdf-files/bueros/fescaribe/15758.pdf

31. Sampieri, Roberto; Carlos Fernández-Collado y Baptista, Pilar. (2006). Metodología de la investigación. México, D. F.: McGraw-Hill.

32. Scott, James. (1990). Los dominados y el arte de la resistencia. Discursos ocultos. México, D. F.: Eras.

33. Soto Alemán, Lien. (2019). Sector privado y contrato económico: reflexiones en el marco de la actualización del modelo económico cubano. Estudios del Desarrollo Social: Cuba y América Latina, 7 (3), pp. 245-257. http://www.revflacso.uh.cu/index. php/EDS/article/view/392

34. Torres, Aylin y Ortega, Diosnara. (2014). Actores económicos y ¿sujetos de la política? La reforma cubana y los trabajadores autónomos. OSAL, 36, pp. 61-80.

35. Torres, Aylin. (2020). Los cuidados al Centro. Una propuesta feminista. Santiago de Chile: Fundación Friedrich Ebert.

[324] 36. Transparency International. (2019). Índice de percepción de la corrupción 2018. https://www.transparency.org/files/content/pages/CPI_2018_Executive_ summary_web_ES.pdf 